\author{
Manuela Fritzsch ${ }^{a}$ \\ Carol Chung Sze Man ${ }^{b}$
}

Die demografische Entwicklung in Europa führt zu einem wachsenden Anteil älterer Menschen in der Gesamtbevölkerung. Mit zunehmendem Alter steigt das Risiko, eine langfristige, chronische Erkrankung zu bekommen. Auch mit einer stetigen Zunahme der Demenzerkrankten ist $\mathrm{zu}$ rechnen. Die Prävalenzrate der Demenz liegt bei den 65- bis 69-Jährigen knapp über $1 \%$, verdoppelt sich dann alle 5 Jahre und erreicht bei Hochaltrigen ab 90 Jahren fast 35\% [1]. Eine WHO-Studie geht davon aus, dass sich die Anzahl der Demenzerkrankten alle 20 Jahre verdoppelt und somit bis 2030 weltweit auf 65,7 Millionen anwächst [2]. Diese Entwicklungen erfordern ein Umdenken im Gesundheitswesen.

Das ganzheitliche Gesundheitskonzept nach Sebastian Kneipp bietet Handlungsansätze, um einerseits das Wohlbefinden der Demenzerkrankten mit einfachen Mitteln zu erhöhen und Befindlichkeitsstörungen zu mindern, damit die Erkrankung für die Betroffenen erträglicher wird. Andererseits bietet es tragfähige Konzepte, um die ständig wachsenden Anforderungen im Pflegebereich zu erfüllen.

\section{Das Kneipp-Gesundheitskonzept}

Die Gesundheitslehre des bayerischen Pfarrers Sebastian Kneipp (18211897) ist ein anerkanntes Naturheilverfahren, das dem europäischen Kulturkreis entstammt. Die Heilkräfte

${ }^{\mathrm{a}} \mathrm{Geschäftsführung,}$

'Öffentlichkeitsarbeit, Kneipp-Bund Landesverband NRW e.V., Essen, Deutschland

\title{
Das Kneipp-Gesundheitskonzept bei demenziell erkrankten Personen
}

der Natur bilden dabei ein wirkungsvolles und nachhaltiges Fundament, das zur Stärkung der Gesundheit mithilfe der fünf Kneipp'schen Elemente Wasser, Bewegung, Ernährung, Heilpflanzen und Lebensordnung beitragen kann. Die Wirkung der Elemente beruht auf dem Reiz-Reaktions-Prinzip, bei dem der Körper auf äussere Reize, die die gewohnten Umwelteinflüsse nach Art und Intensität übersteigen, kurzfristig reagiert. Langfristig bewirkt das regelmässige Training der Körperreaktion einen Anpassungsmechanismus, der zu einer Steigerung der Regulations- und Widerstandsfähigkeit gegenüber auftretenden Reizen führt. Gesundheit entsteht demnach durch die gelungene Balance zwischen der Anpassungsfähigkeit des Organismus und den funktionellen Reizen einer natürlichen Umwelt [3, S. 22].

\section{Die Wasseranwendungen}

Die Kneipp-Wassertherapie umfasst über 120 verschiedene Anwendungen, die man in sieben Kategorien aufteilen kann: Waschungen, Güsse (Tab. 1, Abb. 1), Wickel, Packungen, Dämpfe, Bäder und Abhärtung. Wasseranwendungen setzen durch ihren Temperaturunterschied $\mathrm{zu}$ unserer Körpertemperatur einen gezielten Reiz und führen zu einer stärkeren Durchblutung der Gefässe. Regelmässige Anwendungen haben die Wirkung, die Regulationsfähigkeit unseres Körpers zu stärken und zu stabilisieren. Sie revitalisieren den Organismus und fördern die Immunabwehr. Als Grundsatz gilt die Bedeutung der Reizstärke:

- Kleine Reize entfachen die Lebensfunktionen.
Tab. 1. Knieguss kalt - z.B. bei Krampfadern und beeinträchtigtem venösen Abfluss

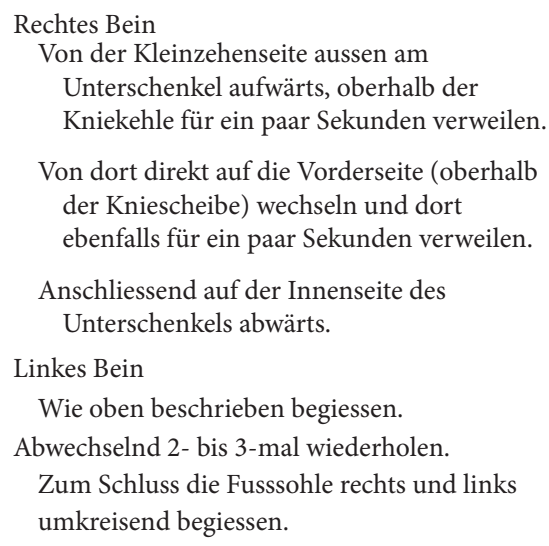

Von dort direkt auf die Vorderseite (oberhalb der Kniescheibe) wechseln und dort ebenfalls für ein paar Sekunden verweilen.

Anschliessend auf der Innenseite des Unterschenkels abwärts.

Linkes Bein

Wie oben beschrieben begiessen. Abwechselnd 2- bis 3-mal wiederholen.

Zum Schluss die Fusssohle rechts und links umkreisend begiessen.

- Gut dosierte, mittlere Reize kräftigen/fördern.

- Übergrosse Reize schaden.

Der zu verarbeitende Temperaturreiz ist für den Organismus umso stärker, je weiter dieser von der Körpertemperatur (ca. $37 \mathrm{Grad}$ ) abweicht, je grösser die behandelte Körperfläche ist und je länger die Anwendung dauert [3, S. 26, 27, 63].

\section{Bewegung}

Die positiven Effekte der Bewegung sind bekannt und wissenschaftlich belegt. Bewegung stabilisiert durch die Ausbildung von Muskeln und Sehnen den Bewegungsapparat und stärkt das Herz-Kreislauf-System. Der Stoffwechsel wird angepasst und die Verdauung reguliert, und der Körper reagiert mit einer geringeren Insulinresistenz. Durch eine vertiefte Atmung findet ein effektiverer Austausch von Stoffen statt. Zudem hat Bewegung einen günstigen Effekt auf das Immunsystem. Wer sich regelmässig bewegt, verbessert die Qualität und Quantität der Immunabwehr-

\section{KARGER}

Fax +497614520714 Information@Karger.com www.karger.com (c) 2014 S. Karger GmbH, Freiburg
Carol Chung Sze Man

Öffentlichkeitsarbeit

Kneipp-Bund Landesverband NRW e.V.

Lindenallee 74, 45127 Essen, Deutschland

c.man@kneippbund-nrw.de

www.kneippbund-nrw.de 
zellen, was zur Senkung einer Infektanfälligkeit führt. Jedoch profitiert nicht nur der Körper von der Bewegung, denn durch den Abbau von Stresshormonen und die Ausschüttung von Glückshormonen hat Bewegung eine ausserordentlich stimmungsaufhellende Wirkung. Menschen, die sich regelmässig bewegen, empfinden mehr Lebensfreude und Lebendigkeit, wodurch das Selbstwertgefühl gesteigert wird. In der Gemeinschaft wirkt sich der soziale Aspekt zusätzlich positiv auf die Gesundheit aus [4].

Allerdings gibt es bisher nur wenig gesichertes Wissen zum Sport mit Hochaltrigen. Unter dem Projekt «NADiA - Neue Aktionsräume für Menschen mit Demenz und ihre Angehörigen» werden aktuell die Effekte eines zweimal pro Woche durchgeführten Trainings, das gemeinsam von demenziell Erkrankten und ihren pflegenden Angehörigen durchgeführt wird, untersucht. Das Projekt wird von Professor Dr. Heinz Mechling des Instituts für Bewegungs- und Sportgerontologie an der Deutschen Sporthochschule (DSHS) in Köln geleitet und stellt ein Folgeprojekt von «fit für 100» (www.ff100.de) dar.

«Fit für 100»: Ziel der zweimal wöchentlich stattfindenden Bewegungsstunden ist eine Verbesserung der Alltagskompetenzen. Das Bewegungsangebot hilft vor allem hochaltrigen und an Demenz erkrankten Menschen, Alltagshandlungen mög-

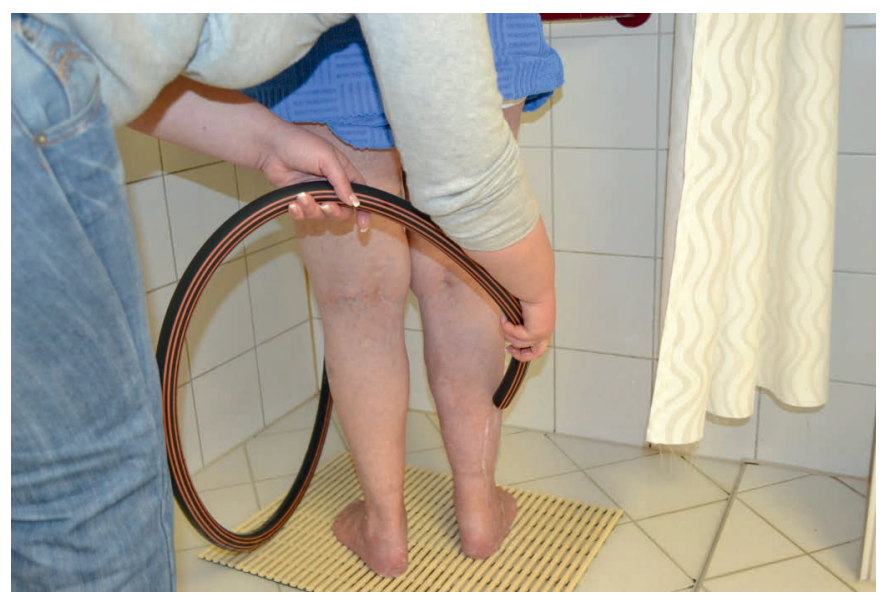

Abb. 1. Knieguss und Wäschung, die in nach Kneipp zertifizierten Senioreneinrichtungen praktiziert werden (Foto:

Kneipp-Bund e.V.). bereits den ausserordentlichen Wert einer gesunden Kostgestaltung erahnen. Er empfahl eine vollwertige Mischkost mit viel Obst, Gemüse, Getreide- und Milchprodukten. Neben der Zusammensetzung ist für eine gesunde Ernährung auch die Art der Nahrungsaufnahme von grosser Bedeutung. Die Mahlzeiten mit allen Sinnen gemeinsam mit anderen $\mathrm{zu}$ geniessen hat einen hohen Stellenwert im Alltag.

Auch Kräuter spielen in der Ernährung eine grosse Rolle. Ätherische Öle in den Kräutern entfalten einen aromatischen Geruch und einen intensiven Geschmack. Sie kurbeln die Säfteproduktion im Körper an und haben eine verdauungsfördernde Wirkung [5].

\section{Heilkräuter}

Kräuter bereichern nicht nur den Speiseplan der Älteren, sondern sie können auch gezielt zur Verbesserung der Lebensqualität und zur Linderung der Begleitsymptome eingesetzt werden. Der intensive Geruch der Kräuter und dadurch ausgelöste Sinneswahrnehmungen bieten neue Zugangswege in die Erinnerungswelt der Erkrankten, die beim Gedächtnistraining und Aktivierungsprogramm des Gehirns sowie in der Biografiearbeit von Nutzen sind.

Der kombinierte Einsatz von Licht, Geräuschen, Tönen und angenehmen Gerüchen wird unter dem Begriff «Snoezelen» zusammengefasst, das in vielen Einrichtungen in einem sogenannten Snoezelraum oder mit einem mobilen Snoezelwagen umgesetzt wird [6]. Die Erfahrung zeigt, dass das Snoezelen Wohlbefinden und Entspannung auslöst; allerdings gibt es keine Studien, die die Wirksamkeit eindeutig belegen.

\section{Lebensordnung}

Ausgehend von ihrem ganzheitlichen Menschenverständnis berücksichtigt die Lebensordnung die körperlichen, gefühlsmässigen und geistigen Aspekte ebenso wie die sozialen 
und spirituellen Dimensionen des menschlichen Lebens. Dieser Begriff beinhaltet alles, was den Ordnungsgesetzen der Natur sowie des Lebens unterliegt und damit unweigerlich Einfluss auf das Leben hat. Um den günstigen Einfluss dieser Gesetze auf die Gesundheit zu nutzen, ist es erforderlich, die Lebensordnung als ganzheitliches Konzept zu verstehen und zu berücksichtigen. Obwohl die Lebensordnung eines der fünf Elemente der Kneipp'schen Lehre darstellt, ist sie doch als übergeordnetes Prinzip gemeint, das die jeweiligen Inhalte miteinander verbindet und in das Leben integriert. Unter einer gelungenen Lebensgestaltung versteht Kneipp eine Einheit zwischen Körper, Geist und Seele, die dabei im Einklang mit dem sozialen und ökologischen Umfeld steht. Ihm ging es darum, dass der Mensch im Einklang mit der Natur und ihren Rhythmen ein sinnerfülltes und zufriedenes Leben führen sollte, indem er umfassende Massnahmen und Bewältigungsstrategien, die ihm zu Gesundheit und gesteigertem Wohlbefinden verhelfen, kennt und diese sinnvoll anzuwenden weiss [7].

\section{Fazit}

Das Pilotprojekt zum Einsatz von Naturheilverfahren in der stationären Altenpflege der Chárite-Universitätsmedizin Berlin in Kooperation mit der Universität Bern, dem Kneipp-Bund e.V. und der Stiftung Zentrum für Qualität und Pflege untersuchte den Gesundheitszustand von pflegebedürftigen Bewohnern und Pflegekräften in den Kneipp-Senioreneinrichtungen. Das Ergebnis zeigt, dass die Einbindung naturheilkundlicher Massnahmen in den Pflegealltag ein Zukunftsmodell für die Versorgung älterer und pflegebedürftiger Menschen sein kann. Die Bewohner waren in einem überdurchschnittlich guten Gesundheitszustand gegenüber Vergleichspopulationen anderer Studien. Zudem konnte die Schmerz- und Beruhigungsmedikation nach der Anwendung von Kneipp-Massnahmen bei den pflegebedürftigen Bewohnern reduziert werden. Auch die Pflegekräfte profitieren hiervon: Das Verhältnis zu den Bewohnern verbesserte sich durch die therapeutischen Massnahmen, der Handlungsspielraum der Arbeit vergrösserte sich, und zufrie- denere Bewohner führten zu einem emotionalen Gewinn der Arbeit.

\section{Literatur}

1 Berlin-Institut für Bevölkerung und Entwicklung: Demenz-Report. Wie sich die Regionen in Deutschland, Österreich und der Schweiz auf die Alterung der Gesellschaft vorbereiten können. Berlin, BerlinInstitut für Bevölkerung und Entwicklung, 2011, p 23.

2 World Health Organization and Alzheimer's Disease International: Dementia: a public health priority. Geneva, WHO, 2012, p 2. www.who.int/mental_health/publications/ dementia_report_2012/en/.

3 Bachmann R, Schleinkofer G: Natürlich gesund mit Kneipp, ed 5. Stuttgart, Trias, 2013.

4 Ahlgrimm C: Seminarhandbuch KneippMentor. Einführung. Essen, Kneipp-Bildungswerk NRW e.V., 2011, p 23.

5 Lenz U: Ernährung; in Kneipp-Bund e.V.: Erfahrung weitergeben - mit Kneipp aktiv und gesund bleiben. Dortmund, Signal Iduna Druck, 2007, pp 12-19.

6 Kitwood T: Demenz. Der personenzentrierte Ansatz im Umgang mit verwirrten Menschen. 1. deutschsprachige Ausgabe von Christian Müller-Hergl. Bern, Hans Huber, 2000, p 89.

7 Paul A: Mind/Body-Medicine, Ordnungstherapie; in Dobos G, Deuse U, Michalsen A (Hrsg): Chronische Erkrankungen integrativ. Konventionelle und komplementäre Therapie. München, Elsevier, 2006, pp 332 333. 